\title{
Aus dem Vorwort zur 1. amerikanischen Auflage
}

Dieses Buch ist als Lehrbuch für die Analysis-Vorlesungen während der ersten beiden Semester des Mathematik- und Physikstudiums gedacht. Abgesehen von einigen Tatsachen über Determinanten, die im Kapitel 9 ohne Beweis angegeben und benutzt werden, sind alle Ergebnisse im Text vollständig hergeleitet. Vom Leser wird allerdings eine gewisse Fähigkeit und Neigung erwartet, abstrakten Gedankengängen zu folgen.

Der erste Teil des Kapitels 1 enthält die Konstruktion der reellen Zahlen mit Hilfe von Schnitten rationaler Zahlen. Dies kann beim ersten Lesen durchaus übergangen werden. Der Rest des Buches kann in diesem Fall logisch dadurch begründet werden, dass man den Dedekindschen Satz postuliert und als Ausgangspunkt wählt. Die Kapitel 1 bis 7 sollten in dieser Reihenfolge durchgegangen werden; die drei letzten Kapitel dagegen sind weitgehend unabhängig voneinander.

Insbesondere für den Anfänger scheint es wichtig, deutlich herauszustellen, dass die Voraussetzungen eines Satzes tatsächlich benötigt werden, um die Gültigkeit seiner Folgerungen zu garantieren. $\mathrm{Zu}$ diesem Zweck ist eine recht große Zahl von Gegenbeispielen in den Text aufgenommen worden.

Jeweils am Ende der Kapitel finden sich Übungsaufgaben, insgesamt über 130 an der Zahl. Einige folgen recht einfach aus den im Text hergeleiteten Ergebnissen, während andere eine Herausforderung an die Originalität der besseren Studenten darstellen. Für die allerschwierigsten sind Hinweise angegeben.

WALTER RUDIN 
